\title{
In vivo two-photon calcium imaging using multi-cell bolus loading
}

\author{
Olga Garaschuk and Arthur Konnerth
}

Physiologisches Institut, Ludwig-Maximilians Universität München, Pettenkoferstr. 12, 80336 München, Germany

Tel: +49-89-5996 584, Fax: +49-89-5996 512 E-mail: olga.garaschuk@Irz.uni-muenchen.de

Goal

This chapter describes an approach for in vivo two-photon $\mathrm{Ca}^{2+}$ imaging of large neuronal circuits with the resolution of individual cells.

\section{Area of application \\ The approach was developed for in vivo imaging of the cortex. It can be easily adapted for imaging other brain regions, including the cerebellum and olfactory bulb. Recently it was successfully used for in vivo recordings from individual spinal cord neurons in zebrafish larvae (Brustein et al., 2003). The staining technique can be also applied in brain slices of any developmental stage, from embryonic to adult.}

\section{Materials}

1. Anesthesia unit including chamber for pre-anesthetic medication and flow meter plus vaporizer (latter items are for volatile anesthetic agents only). Consult literature (for example, Flecknell, 2000) for the best choice of anesthesia for your species. Anesthetic procedures: for adult mice we used either ketamine/xylazine or urethane $(0.1 / 0.01 \mathrm{mg} / \mathrm{g}$ and 1.9 $\mathrm{mg} / \mathrm{g}$ body weight, respectively, i.p.). Alternatively, the mice were anesthetized by inhalation of isoflurane $\left(1.5 \%\right.$ in pure $\left.\mathrm{O}_{2}\right)$.

2. Stereotaxic instrument, drill, warming plate to keep animals body temperature constant (available from many providers, for example, TSE-Systems, Bad Homburg, Germany).

3. Custom-made recording chamber with central access opening (Stosiek et al., 2003).

4. Membrane-permeable calcium indicator dye (e.g. Calcium Green-1 AM, Fura-2 AM, Fluo-4 AM, Indo1 AM, etc.; Molecular Probes, Eugene, Oregon, USA).

5. Manipulator and a pressure application device for injection of the staining solution into the brain. We used the LN-Mini manipulator from Luigs \&Neumann GmbH, Ratingen, Germany and Picospritzer II, General Valve, Fairfield NJ, respectively.

6. Two-photon laser-scanning microscope (see "Twophoton chloride imaging using the quinolinium-based indicator dye MQAE" chapter for details).

\section{Protocol and Procedures}

Staining neurons with a calcium indicator dye

1. Anesthetize the animal. Assure that the surgical level of anesthesia is reached (for example, by testing the pinch withdrawal and the eyelid reflex). Inject $\sim 50$ $\mu \mathrm{l}$ of a local anesthetic agent (e.g. 2\% lidocaine) subcutaneously at the location were the skin is to be removed (optional).

2. Remove the skin above the desired brain area. Perform a small $(\sim 1 \mathrm{~mm})$ craniotomy above an area devoid of big blood vessels. Thin the skull near the craniotomy and polish it with a felt polisher (for example, from Dr. Ihde Dental, Munich, Germany). Use cyanoacryl glue to adhere the custom-made recording chamber to the skull, such that the middle of the chamber opening lies above the craniotomy.

3. Transfer the animal into the set-up and place onto a warming plate $\left(38^{\circ} \mathrm{C}\right)$. Perfuse the recording chamber with a warm $\left(37^{\circ} \mathrm{C}\right)$ standard external saline. We used a saline containing (in $\mathrm{mM}$ ): $125 \mathrm{NaCl}, 2.5 \mathrm{KCl}, 26$ $\mathrm{NaHCO}_{3}, 1.25 \mathrm{NaH}_{2} \mathrm{PO}_{4}, 2 \mathrm{CaCl}_{2}, 1 \mathrm{MgCl}_{2}, 20$ glucose, $\mathrm{pH} 7.4$, when bubbled with $95 \% \mathrm{O}_{2}$ and $5 \% \mathrm{CO}_{2}$.

4. Dissolve acetoxymethyl (AM)-ester of an indicator dye in DMSO plus 20\% Pluronic F-127 (e.g. 2 g Pluronic in $10 \mathrm{ml}$ DMSO) to yield a dye concentration of 10 $\mathrm{mM}$. Dilute this solution $1 / 10$ or $1 / 20$ with the standard pipette solution of the following composition (in $\mathrm{mM}$ ): $150 \mathrm{NaCl}, 2.5 \mathrm{KCl}, 10$ HEPES.

5. Fill a micropipette with this staining solution (pipette resistance 6-9 M $\Omega$ ). Insert the pipette into the cortex and advance it along its axis until it reaches the desired depth (see Fig.1A). Apply a pressure pulse (1 min, 70 $\mathrm{kPa})$ to eject $\sim 400 \mathrm{fl}$ of the staining solution near the cells of interest. Remove the pipette. Wait for an hour to obtain a stable maximal fluorescence level in stained cells (Stosiek et al., 2003). This protocol yields a stained area with a diameter of $200-400 \mu \mathrm{m}$.

Two-photon imaging of stained cells.

We used $800 \mathrm{~nm}$ excitation light to image neurons stained with all indicator dyes tested (see above). The average power under the objective was $<70 \mathrm{~mW}$. With dye application pipette located $150-200 \mu \mathrm{m}$ below the cortical surface, all cortical cells between the surface and $400-\mu \mathrm{m}$ depth are stained (Stosiek et al., 2003). When imaging through the thinned skull (thickness of 8-10 $\mu \mathrm{m}$ ), individual cells could be well resolved up to $200 \mu \mathrm{m}$ below the cortical surface. Removing the skull above the imaging field further improves depth resolution, allowing the detection of individual cells up to $300 \mu \mathrm{m}$ below the cortical surface. It should be stressed that the stability of recordings depends critically on the diameter of the craniotomy. Thus, openings larger than $1 \mathrm{~mm}$ in diameter are often accompanied by movement artefacts occurring at the heartbeat frequency.

\section{Example of application}

Fig. 1B-D shows examples of the in vivo twophoton $\mathrm{Ca}^{2+}$ imaging experiments in the barrel cortex of 
mice (modified from Stosiek et al., 2003). Fig.1B illustrates the quality of imaging data, obtained at different depths. Fig. $1 \mathrm{C}$ shows $\mathrm{Ca}^{2+}$ transients in layer 2/3 neurons evoked by ionophoretic glutamate application in vivo. The glutamate-containing pipette was positioned less then $50 \mu \mathrm{m}$ apart from the imaged cells. $\mathrm{Ca}^{2+}$ transients in Fig. 1D were evoked by the deflection of the majority of whiskers on the contralateral side of the mouse's snout. Note that the signal-to-noise ratio is sufficient to allow individual, non-averaged somatic $\mathrm{Ca}^{2+}$ transients to be distinguished clearly from the background noise.

\section{Advantages and limits}

The approach described, named multi-cell bolus loading (MCBL; Stosiek et al., 2003), allows simultaneous monitoring of $\mathrm{Ca}^{2+}$ levels in many individual neurons. The major difference between MCBL and other staining methods utilizing AM indicator dyes is that the indicators are delivered for a short period directly to the target cells. In particular, this approach improves the staining of neurons in the adult brain, which are, in general, not stained by AM indicator dyes bath-applied to brain slices. Additional advantages of MCBL include the necessity of a minor surgery and the possibility to re-stain neurons and thus to conduct long-lasting, perhaps even chronic (Christie et al., 2001), recordings.

Although MCBL allows to image many cells simultaneously, the resolution of subcellular structures is lower, as compared to in vivo $\mathrm{Ca}^{2+}$ imaging of individual, microelectrode-loaded cells (Svoboda et al., 1997). This is due to two obvious reasons. Firstly, the image contrast is reduced due to the staining of many fine processes in the surrounding neuropil. Secondly, the dye concentration in MCBL-loaded cells is lower, on average $20 \mu \mathrm{M}$ indicator dye, instead of $<3-6 \mathrm{mM}$ when stained using a microelectrode (Stosiek et al., 2003; Svoboda et al., 1997) These limitations restrict the use of MCBL to analyses of somatic $\mathrm{Ca}^{2+}$ transients and make in vivo imaging of neuronal dendrites at present difficult. Furthermore, they reduce the depth resolution of our recordings (200-300 $\mu \mathrm{m}$ compared with $500 \mu \mathrm{m}$ when imaging cell dendrites of microelectrode-loaded cells). Future strategies for improving the quality of recordings include the use of longer wavelengths of the excitation light, larger numerical apertures of the objective lens, better transmittance of the optics, higher photon sensitivity of the PMT, etc. Because the proportion of scattered photons in the emitted fluorescence signal increases markedly with increasing imaging depth, a larger craniotomy and a larger effective angular acceptance of the detection optics (Oheim et al., 2001) should also significantly improve depth resolution by enabling the collection of larger portion of scattered photons.

In conclusion, the approach described here is applicable for $\mathrm{Ca}^{2+}$ imaging of intact neurons both in vivo and in brain slices. It enables staining of adult neurons and, if combined with a miniature head-mounted twophoton microscope (Helmchen et al., 2001), it may allow in vivo two-photon imaging in freely moving animals.

Figure legend.

Fig.1. In vivo $\mathrm{Ca}^{2+}$ imaging of neuronal populations in the barrel cortex of mice. (A) Schematic drawing of the experimental arrangement. (B) High-magnification images of the barrel cortex of a 13-day-old mouse (P13) taken at increasing depth. (C) $\mathrm{Ca}^{2+}$ transients (Lower) in 3 individual layer $2 / 3$ neurons (as indicated in Upper) of another P13 mouse evoked by five consecutive 500-ms ionophoretic glutamate applications. (D) Line-scan recordings of $\mathrm{Ca}^{2+}$ transients (Lower) evoked in two layer $2 / 3$ neurons by a deflection of the majority of whiskers on the contralateral side of the mouse's snout (P13 mouse). The position of the scanned line and the cells analyzed are indicated in Upper.

\author{
References \\ Brustein, E., Marandi, N., Kovalchuk, Y., Drapeau, P. \\ and Konnerth, A. (2003). 'In vivo' monitoring of neuronal \\ network activity in zebrafish by two-photon $\mathrm{Ca}^{2+}$ imaging. \\ Pflugers Arch. in press.
}

Christie, R. H., Bacskai, B. J., Zipfel, W. R., Williams, R. M., Kajdasz, S. T., Webb, W. W. and Hyman, B. T. (2001). Growth arrest of individual senile plaques in a model of Alzheimer's disease observed by in vivo multiphoton microscopy. J. Neurosci. 21, 858-864.

Flecknell, P. (2000). Laboratory animal anaesthesia. San Diego, San Francisco, New York,Boston, London, Sydney, Tokyo: Academic Press.

Helmchen, F., Fee, M. S., Tank, D. W. and Denk, W. (2001). A miniature head-mounted two-photon microscope. high-resolution brain imaging in freely moving animals. Neuron 31, 903-912.

Oheim, M., Beaurepaire, E., Chaigneau, E., Mertz, J. and Charpak, S. (2001). Two-photon microscopy in brain tissue: parameters influencing the imaging depth. $J$ Neurosci. Methods 111, 29-37.

Stosiek, C., Garaschuk, O., Holthoff, K. and Konnerth, A. (2003). In vivo two-photon calcium imaging using multi-cell bolus loading (MCBL). Proc. Natl. Acad. Sci. USA 100, 7319-7324.

Svoboda, K., Denk, W., Kleinfeld, D. and Tank, D. W. (1997). In vivo dendritic calcium dynamics in neocortical pyramidal neurons. Nature 385, 161-165. 